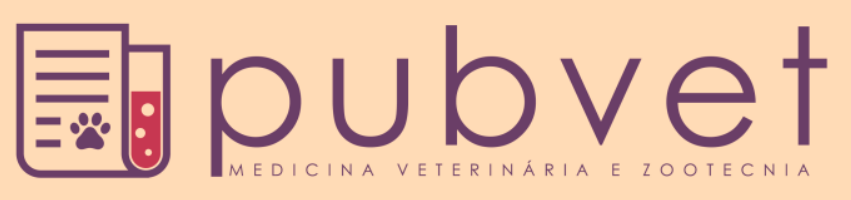

https://doi.org/10.22256/pubvet.v12n6a106.1-6

\title{
Espessura de toucinho e peso de suínos Piau e Duroc utilizando modelos lineares generalizado
}

\author{
Adiel Vieira de Lima $^{1} \bullet$, Leandro Ricardo Rodrigues de Lucena ${ }^{2 *}$, Marco Aurélio \\ Carneiro de Holanda ${ }^{2} \bullet$, Mônica Calixto Ribeiro de Holanda ${ }^{2} \bullet$ \\ ${ }^{I}$ Graduando em Zootecnia, Universidade Federal Rural de Pernambuco, Unidade Acadêmica de Serra Talhada. Serra Talhada -PE Brasil. E- \\ mail: adiel1205@hotmail.com \\ ${ }^{2}$ Professor,Departamento de Zootecnia, Universidade Federal Rural de Pernambuco, Unidade Acadêmica de Serra Talhada. Serra Talhada -PE, \\ Brasil.E-mail: leandroricardo_est@yahoo.com.br, marco.holanda@ufrpe.br, monica.holanda@ufrpe.br. *Autor para correspondência
}

\begin{abstract}
RESUMO. A carne suína está entre as mais antigas formas de alimentação humana, sendo a carne mais consumida no mundo. O Brasil é o $4^{\circ}$ maior produtor e exportador da carne suína no mundo. O principal objetivo de granjas suinícolas industriais é a produção de carcaças com maior teor de carne magra. A redução da espessura de toucinho e da gordura corporal, mais a ampliação da produção de massa magra melhoraram a qualidade da carcaça. O peso vivo de suínos é influenciado pela espessura de toucinho, fazendo com que haja necessidade de encontrar uma relação que explique o comportamento dessas métricas. Para avaliar a relação da espessura do toucinho e o peso vivo dos suínos foi utilizados os modelos lineares generalizados. Os animais foram pesados em balança eletrônica, modelo ID-M 300/4. Para determinação da espessura de toucinho, foram efetuadas mensurações por meio de ultrassonografia pelo equipamento modo A (Lean Meater Renco Model@), a $6 \mathrm{~cm}$ lateralmente à espinha dorsal, entre a penúltima e última costela tomando como resultado a média das avaliações obtidas nos dois lados. Observou-se que para suínos da raça Duroc o modelo linear generalizado com distribuição Gaussiana foi o mais adequado, já para os suínos da raça Piau o mais adequado foi o modelo com distribuição Gamma. Através do modelo uma previsão para espessura de toucinho de um suíno da raça Piau com peso de 100 quilos é em média de 43,31 mm, enquanto que para suínos da raça Duroc esta previsão é de $28,74 \mathrm{~mm}$.
\end{abstract}

Palavras-chave: espessura de toucinho, modelo linear generalizado, suínos

\section{Lard and weight of piau and duroc pigs using generalized linear model}

ABSTRACT. The meat pig is among the oldest forms of human food, being the most consumed meat in the world. Brazil is the 4th largest producer and exporter of meat pig in world. The main objective of industrial pig farms is the production of carcasses with higher lean meat content. Reducing the lard of thickness and body fat plus the increase in lean mass production improved the quality of carcass. The live weight of pigs is influenced by the lard thickness, making it necessary to find a relation that explains the behavior of these metrics. To evaluate the relationship of lard thickness and live weight of pigs, generalized linear models were used. The animals were weighed in an electronic scale, model ID-M 300/4. To determine the lard thickness, measurements were made by ultrasonography using Lean Meater Renco Model ${ }^{\circledR}, 6 \mathrm{~cm}$ side to the spine, between the penultimate and the last rib, resulting in the average of the evaluations obtained in the two sides. It was observed that for Duroc pigs the generalized linear model with Gaussian distribution was the most adequate, whereas for Piau pigs the most suitable was the Gamma distribution model. 
Through the model a forecast for the lard of a pig of the Piau breed weighing $100 \mathrm{~kg}$ is on average $43.31 \mathrm{~mm}$, whereas for Duroc pigs this forecast is $28.74 \mathrm{~mm}$

Keywords: lard, generalized linear model, pigs

\section{Espesor de tocino y peso de cerdos piau y duroc utilizando modelos lineales generalizado}

RESUMEN. La carne de cerdo está entre las más antiguas formas de alimentación humana, siendo la carne más consumida en el mundo. Brasil es el $4^{\circ}$ mayor productor y exportador de la carne de cerdo en el mundo. El principal objetivo de granjas porcinas industriales es la producción de carcasas con mayor contenido de carne magra. La reducción del grosor de tocino y de la grasa corporal, más la ampliación de la producción de masa magra mejoraron la calidad de la carcasa. El peso vivo de cerdos es influenciado por el espesor de tocino, haciendo que haya necesidad de encontrar una relación que explique el comportamiento de esas métricas. Para evaluar la relación del grosor del tocino y el peso vivo de los cerdos se utilizaron los modelos lineales generalizados. Los animales fueron pesados en balanza electrónica, modelo ID-M 300/4. Para la determinación del espesor de tocino, se efectuaron mediciones por ultrasonografía por el equipo modo A (Lean Meater Renco Model®), a $6 \mathrm{~cm}$ lateralmente a la espina dorsal, entre la penúltima y última costilla tomando como resultado la media de las evaluaciones obtenidas en los dos lados. Se observó que para los cerdos de la raza Duroc el modelo lineal generalizado con distribución Gaussiana fue el más adecuado, ya para los cerdos de la raza Piau lo más adecuado fue el modelo con distribución Gamma. A través del modelo una previsión para el espesor de tocino de un cerdo de la raza Piau con peso de 100 kilos es en promedio de 43,31 mm, mientras que para cerdos de la raza Duroc esta previsión es de 28,74 mm.

Palabras clave: espesor de tocino, modelo lineal generalizado, cerdos

\section{Introdução}

A suinocultura mundial vem passando por um intenso melhoramento genético, a fim de selecionando e produzindo suínos de elevado potencial de crescimento, eficiência alimentar e boa composição de carcaça.

O principal objetivo de granjas suinícolas industriais é a produção de carcaças com maior teor de carne magra (Irgang, 1997). Gomes et al. (2008) observaram que a redução da espessura de toucinho e gordura corporal, mais a ampliação da produção de massa magra melhoraram a qualidade da carcaça. Irgang \& Protas (1986) destaca a importância da determinação da espessura de toucinho de suínos reprodutores, observando que para cada milímetro de redução da espessura de toucinho tem-se um ganho de $0,66 \%$ de carne na carcaça.

Costa et al. (2001) afirmam que em programas de melhoramento, para seleção das características de ganho de peso e espessura de toucinho, as correlações genéticas dessas características necessitam de métodos ou procedimentos multivariados. A avaliação da espessura de toucinho é uma ferramenta eficaz no monitoramento da qualidade da carcaça em suínos vivos (Sousa et al., 2010). Segundo Sousa et al. (2010) o peso corporal adequado de matrizes à primeira cobrição é de 125 a $150 \mathrm{~kg}$ e cobertura de gordura que pode variar de 1,1 a 2,2 cm.

De acordo com Rossi et al. (2008) a massa muscular e o peso vivo de porcas gestantes influencia na espessura de toucinho. A espessura de toucinho de porcas lactantes varia de acordo com o peso vivo do animal mais, massa lipídica, peso vivo dos leitões aos sete dias e ganho de peso da leitegada (Rossi et al., 2008).

Diante do exposto o presente trabalho tem como objetivo encontrar uma relação entre a espessura de toucinho e o peso vivos de suínos das raças Piau e Duroc utilizando modelos de regressão lineares generalizados.

\section{Material e Métodos}

A coleta de dados foi realizada no Biotério de Experimentação de Suínos, da Universidade Federal Rural de Pernambuco, Unidade Acadêmica de Serra Talhada. Foram utilizados 43 animais, sendo 18 machos castrados da raça Piau e 25 machos castrados da raça Duroc. Os animais foram alimentados com dietas a base de milho e 
farelo de soja, atendendo às recomendações nutricionais sugeridas por Rostagno et al. (2017).

Os animais foram pesados em uma balança eletrônica, modelo ID-M 300/4, acoplada a uma estrutura de contenção. Para determinação da espessura de toucinho, no momento da pesagem, foram efetuadas mensurações por meio de ultrassonografia usando o equipamento modo A (Lean Meater Renco Model®), a $6 \mathrm{~cm}$ lateralmente à espinha dorsal, entre a penúltima e última costela tomando como resultado a média das avaliações obtidas nos dois lados. No local onde foram realizadas as medidas foi aplicado óleo vegetal em quantidade suficiente para garantir um contato perfeito, sem presença de ar entre a superfície do transdutor e a pele do animal. $\mathrm{O}$ transdutor em posição perpendicular à superfície da pele foi pressionado de maneira suficiente para obter uma leitura constante no visor do aparelho.

Os modelos lineares generalizados foram introduzidos por Nelder \& Wedderburn (1972). Os modelos lineares generalizados apresentam uma característica em que a variável resposta tenha distribuição de probabilidade pertencente à classe de família exponencial. Seja $\mathrm{Y}_{1}, \mathrm{Y}_{2}, \ldots, \mathrm{Y}_{\mathrm{n}}$ variáveis aleatórias independentes, cada uma com função de densidade de probabilidade definida pela seguinte forma:

$\mathrm{f}\left(\mathrm{y}_{\mathrm{i}} ; \theta_{\mathrm{i}} ; \varnothing\right)=\exp \left\{\varnothing\left\{\mathrm{y}_{\mathrm{i}} \theta_{\mathrm{i}}-\mathrm{b}\left(\theta_{\mathrm{i}}\right)\right\}+\mathrm{c}\left(\mathrm{y}_{\mathrm{i}} ; \varnothing\right)\right\}(1)$

Se $Y_{i}$ apresenta a forma acima denominamos de classe de família exponencial. Pode-se mostrar sob as condições usuais de regularidade

$$
\mathrm{E}\left\{\frac{\delta \operatorname{logf}\left(\mathrm{Y}_{\mathrm{i}} ; \theta_{\mathrm{i}} ; \varnothing\right)}{\delta \theta_{\mathrm{i}}}\right\}=0
$$

$\mathrm{e}$

$$
\mathrm{E}\left\{\frac{\delta^{2} \operatorname{logf}\left(\mathrm{Y}_{\mathrm{i}} ; \theta_{\mathrm{i} ;}, \varnothing\right)}{\delta \theta_{\mathrm{i}}{ }^{2}}\right\}=-\mathrm{E}\left[\left\{\frac{\delta \operatorname{logf}\left(\mathrm{Y}_{\mathrm{i}} ; \theta_{\mathrm{i} ;}, \boldsymbol{}\right)}{\delta \theta_{\mathrm{i}}}\right\}\right]^{2}
$$

onde, $\quad \mathrm{E}\left(\mathrm{Y}_{\mathrm{i}}\right)=\mu_{\mathrm{i}}=\mathrm{b}^{\prime}\left(\theta_{\mathrm{i}}\right)$ e $\operatorname{Var}\left(\mathrm{Y}_{\mathrm{i}}\right)=$ $\emptyset^{-1} \mathrm{~V}\left(\mu_{\mathrm{i}}\right)$, em que $\mathrm{V}\left(\mu_{\mathrm{i}}\right)=\frac{\mathrm{d} \mu_{\mathrm{i}}}{\mathrm{d} \theta_{\mathrm{i}}}$ é a função de variância e $\emptyset^{-1}>0(\varnothing>0)$ é o parâmetro de dispersão.
Os modelos lineares generalizados são definidos pela equação (1) e pela parte sistemática

$$
\mathrm{g}\left(\mu_{\mathrm{i}}\right)=\eta_{\mathrm{i}}
$$

onde, $\eta_{i}=X_{i}^{\prime} \beta$ é o preditor linear, $\beta=$ $\left(\beta_{1}, \beta_{2}, \ldots, \beta_{\mathrm{p}}\right)^{\prime}, \mathrm{p}<\mathrm{n}$, é um vetor de parâmetros desconhecidos a serem estimados, $\mathrm{X}_{\mathrm{i}}=$ $\left(\mathrm{x}_{\mathrm{i} 1}, \mathrm{x}_{\mathrm{i} 2}, \ldots, \mathrm{x}_{\mathrm{ip}}\right)^{\prime}$ representa os valores das variáveis explicativas e $\mathrm{g}($.) é uma função monótona e diferenciável, denominada função de ligação (Paula, 2004).

A estimação dos parâmetros em um modelo linear generalizado é definida pela maximização da função de log-verossimilhança, que é o logaritmo do produtório da função de densidade de probabilidade definida na equação (1), ou seja,

$$
\begin{gathered}
\mathrm{L}=\sum_{\mathrm{i}=1}^{\mathrm{n}} \log \mathrm{f}\left(\mathrm{Y}_{\mathrm{i}} ; \theta_{\mathrm{i}} ; \varnothing\right)=\sum_{\mathrm{i}=1}^{\mathrm{n}}\left\{\varnothing \left\{\mathrm{y}_{\mathrm{i}} \theta_{\mathrm{i}}-\right.\right. \\
\left.\left.\mathrm{b}\left(\theta_{\mathrm{i}}\right)\right\}+\mathrm{c}\left(\mathrm{y}_{\mathrm{i}} ; \varnothing\right)\right\}(2)
\end{gathered}
$$

Porém, a solução dessa maximização desta função depende da utilização de métodos numéricos, como o método de Newton-Raphson (Paula, 2004). Aplicando o método de NewtonRaphson tem-se que a estimação dos parâmetros é definida por:

$$
\widehat{\beta}^{\mathrm{m}+1}=\left(\mathrm{X}^{\prime} \mathrm{W}^{\mathrm{m}} \mathrm{X}\right)^{-1} \mathrm{X}^{\prime \mathrm{W}^{\mathrm{m}} \mathrm{z}^{\mathrm{m}}}
$$

em que, $\mathrm{m}=0,1,2, \ldots$ é o passo iterativo, $\widehat{\beta}^{\mathrm{m}+1}$ é a estimava dos parâmetros na iteração $\mathrm{m}, \mathrm{X}$ é a matriz dos valores da variáveis explicativas, $\mathrm{W}$ é a matriz de peso que muda a cada passo do processo iterativo e z é o vetor ajustado da variável dependente na m-ésima iteração (Paula, 2004).

A qualidade do ajuste de um modelo linear generalizado é avaliada através da função desvio definida por:

$$
\begin{gathered}
\mathrm{D}(\mathrm{y} ; \hat{\mu})=2 \sum_{\mathrm{i}=1}^{\mathrm{n}}\left\{\mathrm{y}_{\mathrm{i}}\left(\check{\theta}_{\mathrm{i}}-\widehat{\theta}_{\mathrm{i}}\right)+\left(\mathrm{b}\left(\widehat{\theta}_{\mathrm{i}}\right)-\right.\right. \\
\left.\left.\mathrm{b}\left(\check{\theta}_{\mathrm{i}}\right)\right)\right\}(4)
\end{gathered}
$$

onde, $\hat{\theta}_{\mathrm{i}}=\theta_{\mathrm{i}}\left(\hat{\mu}_{\mathrm{i}}\right)$ e $\check{\theta}_{\mathrm{i}}=\theta_{\mathrm{i}}\left(\check{\mu}_{\mathrm{i}}\right)$ são as estimativas de máxima verossimilhança de $\theta$ para os modelos com p parâmetros $(\mathrm{p}<\mathrm{n})$ e saturado $(\mathrm{p}$ $=n$ ), respectivamente. Tem-se ainda que $\hat{\mu}_{\mathrm{i}}=$ $\mathrm{g}^{-1}\left(\hat{\eta}_{\mathrm{i}}\right)$ onde $\hat{\eta}_{\mathrm{i}}=\mathrm{X}_{\mathrm{i}}^{\prime} \hat{\beta}$ e $\breve{\mu}_{\mathrm{i}}=\mathrm{y}_{\mathrm{i}}$.

Para avaliar a adequação do modelo linear generalizado foi utilizado a soma dos quadrados dos resíduos (SQR), o coeficiente de determinação do modelo $\left(\mathrm{R}^{2}\right)$, o critério de informação de 
Akaike (Akaike, 1974) e o índice d de Willmott (Willmott, 1981).

A soma dos quadrados dos resíduos (SQR) é expressa por:

$$
S Q R=\sum_{i=1}^{n}\left(Y_{i}-\widehat{Y}_{1}\right)^{2}
$$

O coeficiente de determinação do modelo é expresso pela razão entre a soma de quadrado do modelo (SQM) e a soma de quadrados total (SQT), ou seja,

$$
\mathrm{R}^{2}=\frac{\mathrm{SQM}}{\mathrm{SQT}}=1-\frac{\mathrm{SQR}}{\mathrm{SQT}}=1-\frac{\sum_{\mathrm{i}=1}^{\mathrm{n}}\left(\mathrm{Y}_{\mathrm{i}}-\widehat{\mathrm{Y}}_{1}\right)^{2}}{\sum_{\mathrm{i}=1}^{\mathrm{n}}\left(\mathrm{Y}_{\mathrm{i}}-\overline{\mathrm{Y}_{1}}\right)^{2}}
$$

O critério de informação de Akaike (AIC) é definido por:

$$
\mathrm{AIC}=\mathrm{n} \log \left\{\frac{\mathrm{D}(\mathrm{y} ; \hat{\mu})}{\mathrm{n}}\right\}+2 \mathrm{p}
$$

onde, $\mathrm{D}(\mathrm{y} ; \hat{\mu})=\sum_{\mathrm{i}=1}^{\mathrm{n}}\left(\mathrm{Y}_{\mathrm{i}}-\widehat{\mathrm{Y}}_{1}\right)^{2}$.

O índice d é definido por Willmott (1981) é dado por:

$$
\mathrm{d}=1-\frac{\sum_{\mathrm{i}=1}^{\mathrm{n}}\left(\widehat{\mathrm{Y}}_{1}-\mathrm{Y}_{\mathrm{i}}\right)^{2}}{\sum_{\mathrm{i}=1}^{\mathrm{n}}\left(\left|\widehat{\mathrm{Y}}_{1}-\overline{\mathrm{Y}}\right|+\left|\mathrm{Y}_{\mathrm{i}}-\overline{\mathrm{Y}}\right|\right)^{2}}
$$

onde, $\bar{Y}$ é a media dos valores da espessura de toucinho dos suínos (Yi).

Seja Y uma variável aleatória com distribuição gamma de média $\mu$ e coeficiente de variação $\emptyset^{-1 / 2}$, denotamos $Y \sim G(\mu, \emptyset)$ cuja função de densidade de probabilidade é expressa por:

$$
f\left(y_{i} ; \theta_{i ;} \varnothing\right)=\frac{1}{\Gamma(\phi)}\left(\frac{\phi y}{\mu}\right)^{\phi} \exp \left(-\frac{\phi y}{\mu}\right) d(\log y)
$$

logo, a função de densidade de probabilidade da gamma na forma da família exponencial é dada por:

$$
\begin{aligned}
\mathrm{f}\left(\mathrm{y}_{\mathrm{i}} ; \theta_{\mathrm{i}} ; \varnothing\right)= & \exp \left[\phi\left\{\left(\frac{-\mathrm{y}}{\mu}\right)-\log \mu\right\}-\log \Gamma(\phi)+\right. \\
& \phi \log (\phi y)-\log \mathrm{y}](6)
\end{aligned}
$$

onde, $y>0, \phi>0, \mu>0 \quad$ e a função $\Gamma(\phi)$ é dada por:

$$
\Gamma(\phi)=\int_{0}^{\infty} t^{\emptyset-1} e^{-t} d t
$$

Utilizando a integração por partes, tem-se que $\Gamma(\phi)=(\varnothing-1)$ !

Sejam $\mathrm{Y}_{1}, \mathrm{Y}_{2}, \ldots, \mathrm{Y}_{\mathrm{n}}$ variáveis aleatórias independentes tais que $\mathrm{Y}_{\mathrm{i}} \sim \mathrm{G}\left(\mu_{\mathrm{i}}, \varnothing\right)$. Suponha ainda que $g\left(\mu_{\mathrm{i}}\right)=\eta_{\mathrm{i}}$ com $\eta_{\mathrm{i}}=\mathrm{X}_{\mathrm{i}}^{\prime} \beta, \mathrm{X}_{\mathrm{i}}=$ $\left(\mathrm{x}_{\mathrm{i} 1}, \mathrm{x}_{\mathrm{i} 2}, \ldots, \mathrm{x}_{\mathrm{ip}}\right)^{\prime}$, um vetor com valores das variáveis explicativas e $\beta=\left(\beta_{1}, \beta_{2}, \ldots, \beta_{p}\right)^{\prime}$ o vetor de parâmetros. Utilizando a função de ligação $\mu_{\mathrm{i}}=\eta_{\mathrm{i}}$ tem-se que a estimativa dos parâmetros $\beta$ é dado por:

$$
\beta^{m+1}=\left(X^{\prime} W^{m} X\right)^{-1} X^{\prime} W^{m} z^{m}
$$

onde, $\mathrm{m}=0,1, \ldots$ é o passo da iteração, $\mathrm{z}=\eta+$ $W^{-\frac{1}{2}} V^{-\frac{1}{2}}(y-\mu), \eta=\left(\eta_{1}, \eta_{2}, \ldots, \eta_{n}\right)^{\prime}, y=$ $\left(\mathrm{y}_{1}, \mathrm{y}_{2}, \ldots, \mathrm{y}_{\mathrm{n}}\right)^{\prime}, \mu=\left(\mu_{1}, \mu_{2}, \ldots, \mu_{\mathrm{n}}\right)^{\prime}, \mathrm{V}=$ $\operatorname{diag}\left\{\mu_{1}, \mu_{2}, \ldots, \mu_{n}\right\}, W=\operatorname{diag}\left\{\mathrm{w}_{1}, \mathrm{w}_{2}, \ldots, \mathrm{w}_{\mathrm{n}}\right\}$, $\mathrm{w}_{\mathrm{i}}=\frac{\left(\frac{\mathrm{d} \mu_{\mathrm{i}}}{\mathrm{d} \eta_{\mathrm{i}}}\right)^{2}}{\mu_{\mathrm{i}}}$

Seja Y uma variável aleatória com distribuição normal de média $\mu$ e variância $\sigma^{2}$, denotamos $\mathrm{Y}$ $\mathrm{N}\left(\mu, \sigma^{2}\right)$ cuja função de densidade de probabilidade é expressa por:

$$
\mathrm{f}\left(\mathrm{y}_{\mathrm{i}} ; \mu_{\mathrm{i}} ; \sigma^{2}\right)=\frac{1}{\sigma \sqrt{2 \pi}} \exp \left(-\frac{1}{2 \sigma^{2}}(\mathrm{y}-\mu)^{2}\right)
$$

logo, a função de densidade de probabilidade da normal na forma da família exponencial é dada por:

$$
\begin{gathered}
\mathrm{f}\left(\mathrm{y}_{\mathrm{i}} ; \mu_{\mathrm{i}} ; \sigma^{2}\right)=\exp \left[\left\{\frac{1}{\sigma^{2}}\left(\mu \mathrm{y}-\frac{\mu^{2}}{2}\right)-\right.\right. \\
\left.\left.\frac{1}{2}\left\{\log 2 \pi \sigma^{2}+\frac{\mathrm{y}^{2}}{\sigma^{2}}\right\}\right\}\right]
\end{gathered}
$$

em que $-\infty<\mu, \mathrm{y}<\infty$ e $\sigma^{2}>0$. O processo de estimação dos parâmetros segue o mesmo da distribuição gamma.

Para ambas as raças foram ajustados os modelos de regressão com distribuição Gamma e Gaussiana como descritos na Tabela 1, porém nos resultados são apresentados apenas os modelos com melhor ajuste. 
Tabela 1. Modelos de regressão avaliados para suínos das raças Piau e Duroc.

\begin{tabular}{lc}
\hline $\begin{array}{l}\text { Modelos de } \\
\text { Regressão }\end{array}$ & Equação de Regressão \\
\hline \multicolumn{3}{c}{ Piamma } & $\mathrm{Y}_{\mathrm{i}}=\beta_{0}+\beta_{1}$ Peso $_{i}+\varepsilon_{i}$ \\
Gaussiano & $\mathrm{Y}_{\mathrm{i}}=\beta_{0}+\beta_{1}$ Peso $_{i}+\varepsilon_{i}$ \\
& Duroc \\
Gamma & $\mathrm{Y}_{\mathrm{i}}=\beta_{0}+\beta_{1}$ Peso $_{i}+\varepsilon_{i}$ \\
Gaussiano & $\mathrm{Y}_{\mathrm{i}}=\beta_{0}+\beta_{1}$ Peso $_{i}+\varepsilon_{i}$ \\
\hline
\end{tabular}

onde, $Y_{i}$ é a i-ésima espessura de toucinho do suíno da raça Piau ou Duroc, Pesoi é o peso do iésimo suíno avaliado e $\varepsilon_{\mathrm{i}}$ é o i-ésimo erro associado a espessura de toucinho do animal, em que apresenta distribuição normal de média 0 e variância constante $\sigma^{2}$ para o modelo Gaussiano, e distribuição Gamma de parâmetros $\alpha$ e $\beta$ para o modelo gamma. As incógnitas $\beta_{0}$ e $\beta_{1}$ são os parâmetros associados ao modelo.

\section{Resultados e Discussão}

Verifica-se na Figura 1 a relação entre a espessura de toucinho e o peso dos suínos da raça Piau, nota-se que à medida que o peso aumenta a espessura de toucinho também aumenta indicando uma correlação do tipo positiva. Observa-se ainda que os valores ajustados pelo modelo estão bem próximos dos observados. Observa-se ainda que através do modelo uma previsão para espessura de toucinho de um suíno da raça Piau com peso de 100 quilos é em média de 43,31 mm.

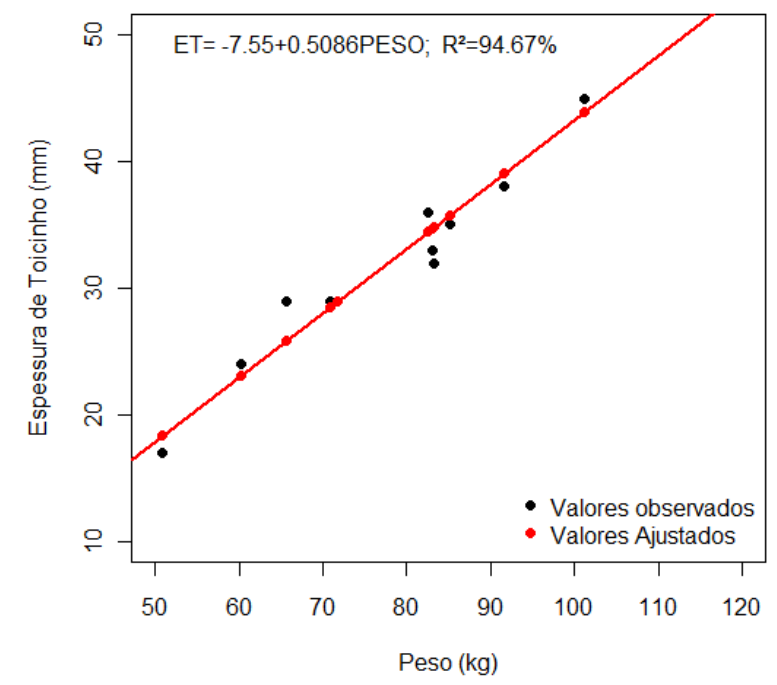

Figura 1. Relação entre a espessura de toucinho (ET) e o peso de suínos da raça Piau.

O modelo ajustado para explicar a relação entre a espessura de toucinho e o peso dos suínos da raça Piau foi o modelo de distribuição Gamma, Tabela 2. Observa-se que o modelo apresentou um poder de explicação superior a 94\%, baixa soma de quadrados de resíduos 29,06 e $\mathrm{AIC}=49,15$, e alto valor do índice de Willmott (d=0,9866), Tabela 2.

Tabela 2. Modelo ajustado e critérios de adequação do modelo para relação entre espessura de toucinho (ET) e o peso vivo dos suínos.

\begin{tabular}{lcccc}
\hline Modelo Ajustado & \multicolumn{5}{c}{ Critérios de Adequação do Modelo } \\
& $\mathrm{R}^{2}$ & AIC & SQR & d \\
\hline \multirow{3}{*}{ ET $=-7,55+0,5086$ Peso } & Piau & & & \\
& 94,67 & 49,15 & 29,06 & 0,9866 \\
ET $=0.2874$ Peso & Duroc & & & \\
& 99,80 & 10,22 & 24,40 & 0,9745 \\
\hline
\end{tabular}

Verifica-se na Figura 2 a relação entre a espessura de toucinho e o peso dos suínos da raça Duroc, nota-se que à medida que o peso aumenta a espessura de toucinho também aumenta indicando uma correlação do tipo positiva. Observa-se que o modelo apresentou bons ajustes para maioria das espessuras de toucinhos observadas. Através do modelo ajustado uma previsão para espessura de toucinho de um suíno da raça Duroc com 100 quilos é em média de 28,74 $\mathrm{mm}$. O modelo ajustado para explicar a relação entre a espessura de toucinho e o peso dos suínos da raça Duroc foi o modelo de distribuição normal, Tabela 2. Observa-se que o modelo apresentou um alto poder de explicação $99,80 \%$, soma de quadrados de resíduos 24,40, AIC de 10,21 e alto índice de Willmott (d=0,9745), Tabela 2.

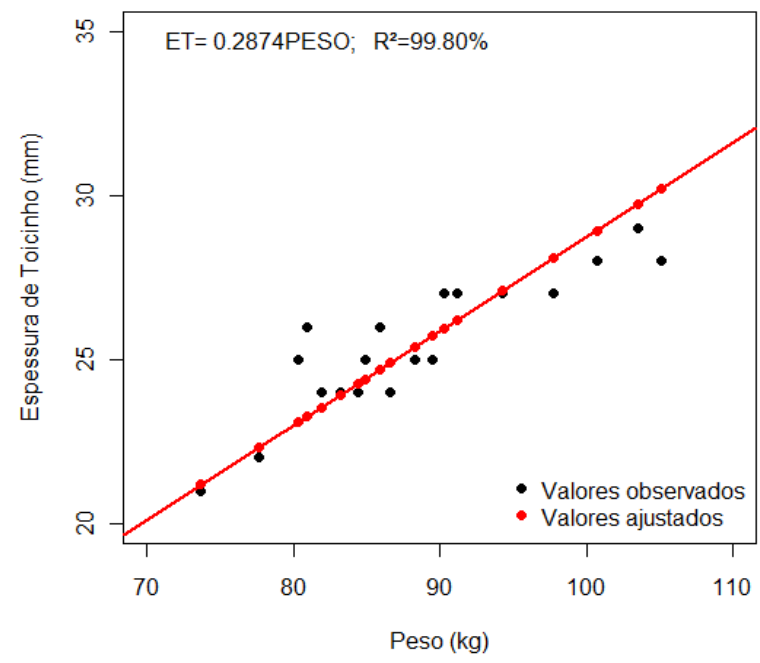

Figura 2. Relação entre a espessura de toucinho (ET) e o peso de suínos da raça Duroc.

Através da modelagem matemática pelos modelos lineares generalizados foi possível definir uma relação entre a espessura do toucinho e o peso vivo dos suínos das raças Piau e Duroc. Para suínos da raça Piau a relação foi expressa 
com ajuste do modelo Gamma, já para os suínos da raça Duroc a relação foi definida pelo modelo foi o Gaussiano.

\section{Referências bibliográficas}

Akaike, H. 1974. A new look at the statistical model identication. IEEE Transactions on automatic Control, 19(6), 716-723.

Costa, A. R. C., Lopes, P. S., Torres, R. A., Regazzi, A. J., Silva, M. A., Euclydes, R. F. \& Pires, A. V. 2001. Estimação de parâmetros genéticos em características de desempenho de suínos das raças Large White, Landrace e duroc. Revista Brasileira de Zootecnia, 30(1), 49-55.

Gomes, J. D. F., Putrino, S. M., Martelli, M. R., Sobral, P. J. A. \& Fukushima, R. S. 2008. Desempenho e características de carcaça de suínos alimentados com dieta com feno de tifton (Cynodon Dactylon). Ciência Animal Brasileira, 9(1), 59-67.

Irgang, R. 1997. A Força da genética. Suinocultura Industrial, 23, 26-27.

Irgang, R. \& Prostas, J. F. S. 1986. Peso ótimo para abate de suínos. Pesquisa Agropecuária Brasileira, 21(12), 1337-1345.

Nelder, J. A. \& Wedderburn, R. W. 1972. Generalized linear models. Journal of the
Royal Statistical Society, Series A, 135(3), 370-384.

Paula, G. A. 2004. Modelo de Regressão com Apoio Computacional. IMEUSO, São Paulo.

Rossi, C. A. R., Lovatto, P. A., Weschenfelder, V. A. \& Lehnen, C. R. 2008. Metanálise da relação entre espessura de toicinho e variáveis corporais e reprodutivas de porcas gestantes e lactantes. Ciência Rural, 38(1), 206-212.

Rostagno, H. S. 2017. Tabelas brasileiras para aves e suínos: composição de alimentos $e$ exigências nutricionais (4a ed.). ISE-MG, Viçosa.

Sousa, F. A. D., Silva, M. A. P., Corrêa, F. R., Leão, K. M. \& Guimarães, T. P. 2010. Correlação entre os parâmetros de peso vivo, idade e espessura de toucinho em suínos. PUBVET, 4(41), 1-6.

Willmott, C. J. 1981. On the validation of models. Physical Geography, 2(2):184-194.

Article History:

Received 16 April 2018

Accepted 2 May 2018

Available online 31 May 2018

License information: This is an open-access article distributed under the terms of the Creative Commons Attribution License 4.0, which permits unrestricted use, distribution, and reproduction in any medium, provided the original work is properly cited. 